\title{
3D RECONSTRUCTION OF VASCULAR STRUCTURES USING GRAPH-BASED VOXEL COLORING
}

\author{
Rémi Martin * Étienne Vachon * Joaquim Miró ${ }^{\dagger} \quad$ Luc Duong * \\ * Ecole de technologie supérieure \\ Department of Software and IT Engineering \\ 1100 Notre-Dame W., Montréal, Canada. \\ $\dagger$ Department of Pediatrics, CHU Sainte-Justine \\ 3175 Cote-Ste-Catherine, Montréal, Canada
}

\begin{abstract}
Biplane X-ray angiography is currently the gold standard for navigational guidance during percutaneous interventions in vascular structures; but it remains limited to $2 \mathrm{D}$ projections. In this study, we propose a novel graph-based voxel coloring method for 3D reconstruction of vascular structures from biplane X-ray angiography sequences. The reconstruction is obtained by using the random walks algorithm on a graphbased representation of a discretized visual hull, to obtain the probability of belonging to the vascular structure. A multiscale scheme is introduced to reconstruct at a finer level, while being computationally efficient. The proposed method was validated using the XCAT motion simulator and on calibrated clinical data.
\end{abstract}

Index Terms - X-ray angiography, 3D reconstruction, Random walks, vascular structures, biplane reconstruction.

\section{INTRODUCTION}

X-ray angiography is still considered the gold standard for navigation during percutaneous interventions. To visualize soft tissues, the cardiologist must inject a contrast agent in the targeted vessels. As a 2D representation of a 3D object, X-ray angiography suffers from foreshortening and requires expert cardiologists to mentally reconstruct both views in the patient's reference frame. Providing an online 3D model without requiring more images than those already obtained during intervention would provide cardiologists with a useful and convenient view of the patient's anatomy.

Using only two views for 3D reconstruction is a challenging problem that requires solving more ambiguities than in three or more views, especially where overlapping can occur. Hadida et al. have proposed a stochastic motion compensation scheme using a single view with a preoperative CT [1]. The use of the fewer possible views to perform the reconstruction is a great benefit considering the exposition of

This research was funded by NSERC Discovery grant. the patients to X-rays and contrast agents. Obtaining a 3D reconstruction directly with biplane $\mathrm{X}$-ray angiography used routinely during the intervention might contribute to reduce the amount of exposition to radiation and contrast agent compared to, for instance, a single-plane rotational acquisition. Moreover, the reconstructed anatomical structure will be in the same reference than the patient.

Multiview voxel-based 3D reconstruction has been a prolific field of study [2]. Popular approaches such as voxel coloring [3], space carving and volume intersection were proposed to solve this problem. However, since vascular structures are elongated and nearly cylindrical in nature, those techniques may not produce a clinically useful reconstruction. Most studies about 3D reconstruction, such as [4], assume that vascular structures are of an ellipsoidal form, but irregularities of vascular structures like stenosis or aneurysm may not be fully represented. Multiview reconstruction methods, such as shape from silhouette, were proposed for the 3D reconstruction of coronary arteries for such difficult cases [5]. Random walks are widely popular for image segmentation [6], in 2D as well as in 3D, for volumetric image segmentation in CT and MRI. To our knowledge, this is the first study to solve the voxel coloring problem using Random Walks and to perform a 3D reconstruction of a biplane X-ray angiography sequence. Hence, the contributions of this study are as follows: 1) to reconstruct large vascular structures in $3 \mathrm{D}$, such as the aorta or pulmonary artery, $\mathrm{S} 2$ ) to introduce a random walks formulation to solve the voxel coloring problem, 3) to validate on simulated and patient data.

\section{METHOD}

The proposed approach is divided in four major steps: preprocessing of the images, graph construction, volume reconstruction and finally, multiscale refinement. This process is repeated for each image in the sequence to achieve a 3D reconstruction at each pair of biplane image of the X-ray angiography sequence. 
Although, even if it is well known that both projections in biplane angiography systems are not precisely acquired at the same time, we consider that it is negligible in the method below. Views were calibrated using the parameters contained in the DICOM headers, and with a calibration procedure using the guide wire, as presented by Vachon et al. [7].

\subsection{Graph construction}

The first step of our methodology is to segment the artery in both images used for the 3D reconstruction. This step is performed using multiscale vesselness filtering [8] and manual editing to refine the resulting segmentation that might not be good enough on large vascular structures. Second, the voxelbased reconstruction is conducted by defining an energy function over a 3D discrete lattice and by optimizing it. A vertex $i$ in our graph corresponds to a voxel and is connected to the vertices in its neighborhood $N_{i}$. For the vertices that are not located at the boundary of the volume, the size of $N_{i}$ is 26 . Let $e_{i, j}$ denote the edge between vertices $i$ and $j$. We associate a weight $w_{i, j}$ to the edge $e_{i, j}$ defined as:

$$
w_{i, j}=\exp \left(-\alpha\left(b_{i}-b_{j}\right)^{2}\right)
$$

where $\alpha$ is used to penalize small differences. We also consider that for a vertex $i$ :

$$
b_{i}=\frac{b_{P_{i, 1}}+b_{P_{i, 2}}}{2}
$$

Where $P_{i, p l}$ represents the projection of the vertex $i$ on the plane $p l \in\{1,2\}$. Binary values $b_{P_{i, p l}}$ are computed using the segmentation of the images.

We also consider $d_{i}$ to represent the ratio between the minimal 3D distance and the minimal 2D distance of the vertex $i$ and the centerline, both in $\mathrm{mm}$, as follows:

$$
d_{i}=\frac{\min \left(d_{2 D, i}, d_{3 D, i}\right)}{\max \left(d_{2 D, i}, d_{3 D, i}\right)}
$$

The 2D distance, $d_{2 D, i}$, is obtained by calculating the 2D distance of the projection of a vertex $i$ with the centerline on each view and by selecting the maximum among those two. The 3D centerline of the artery is calculated using the epipolar geometry [9]. Using the two views, the manually identified correspondences between the pre-extracted centerlines are known. Then, a triangulation is performed using the pair of $2 \mathrm{D}$ points to obtain the $3 \mathrm{D}$ points composing the $3 \mathrm{D}$ centerline. Using a manual pairing of the points in correspondence allows us to solve any ambiguity that might occur using the epipolar geometry.

\subsection{Volume reconstruction}

The voxel value, $b_{i}$, of each vertex provides a first estimate of the 3D model that contains the final volume. This information, on its own, is not sufficient to outline the vascular

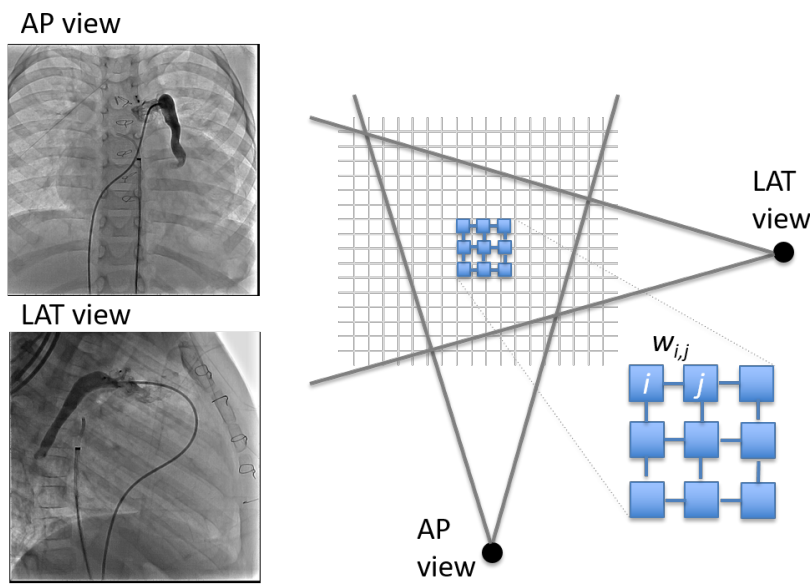

Fig. 1. Voxel coloring using random walks. A visual hull is extracted from 3D space. A graph is constructed from the convex hull. Each voxel is modeled as a node in a graph.

structure. A spatial constraint such as the distance to the 3D centerline is introduced to ensure that the voxels closer to the centerline will be favored over voxels farther to the vascular structure.

Once all the weights $w_{i, j}$ are computed, a mapping function $f$ is defined to identify all the vertices that belong to the artery and exclude those that compose the background. This function assigns a probability value to each vertex of the graph. If the $i-t h$ vertex of the graph is part of the artery then $f_{i}=1$ otherwise $f_{i}=0$. We aim to minimize the following energy function:

$$
\begin{aligned}
E(f)= & \frac{1}{2} \sum_{i=1}^{N} \sum_{j=1}^{N} w_{i, j}\left(f_{i}-f_{j}\right)^{2} \\
& +\beta \sum_{1=i}^{N}\left(1-d_{i}\right) f_{i}^{2} \\
& +\gamma \sum_{1=i}^{N} d_{i}\left(f_{i}-1\right)^{2}
\end{aligned}
$$

The first term of the equation is used to link together sections of the same vascular structure by minimizing the weight of the edges at the boundary of the artery (i.e. cut edges). The second one minimizes the distance ratio of the vertices of the background, while the third one maximizes the distance ratio of the artery. By using the distance ratio we ensure a certain coherence between what is seen on the 2D images and the final model. In other terms, if the 2D and 3D distances differ greatly, then the corresponding voxel is not selected. In order to obtain the values of $f$, we define the energy function in a matrix form. We denote $L_{w}$ as the Laplacian matrix of the weights of our graph, $I$ as the identity matrix and $D$ the 
diagonal matrix of d such that $D_{i i}=d_{i}$.

$$
E(f)=f^{T}\left(L_{w}+\beta I+(\gamma-\beta) D\right) f-2 \gamma d^{T} f+\gamma d^{T} \mathbb{1}
$$

By differentiating the previous equation with respect to $f$ and by setting $\gamma=\beta$, in a similar manner to [10], we obtain:

$$
f=\beta\left(L_{w}+\beta I\right)^{-1} d
$$

The constant $\beta$ can be used to alter the weight between the binary value and the distance ratio.

\subsection{Multiscale volume refinement}

The reconstruction process described above, provides a coarse volumetric model of the vascular structure. To enhance the accuracy of the result, we perform a multi-scale refinement. This step consists in dividing the size of the vertices of low probability. The idea is to prevent re-running the entire algorithm with the new divided vertices and to just calculate the binary value of newly created vertices. A threshold is applied and the vertices with a binary value greater than a constant, $A$, will be added to the final volume. The vertices smaller are divided again until a pre-defined number of steps for the multi-scale is reached.

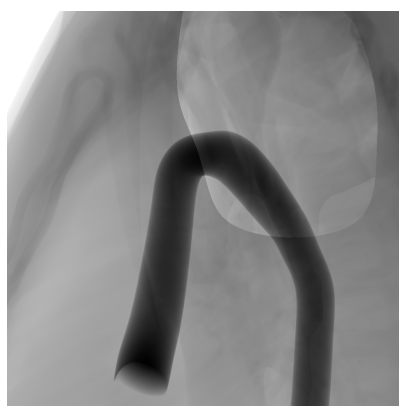

(a) Simulated image - LAT

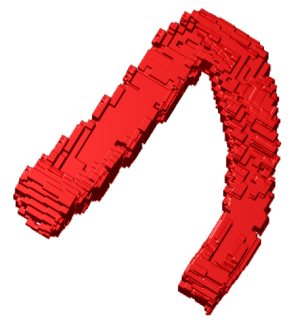

(c) 3D model - LAT

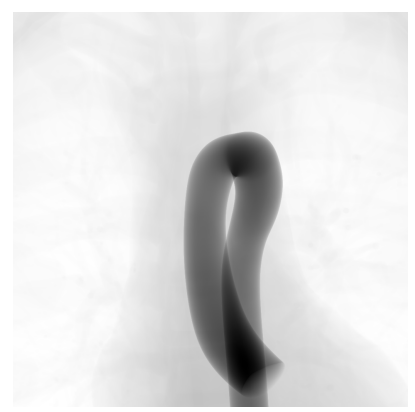

(b) Simulated image - PA

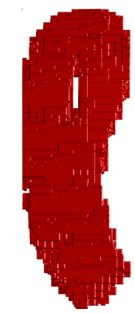

(d) 3D model - PA
Fig. 2. 3D model of the reconstructed aorta along with the images used to perform the reconstruction.

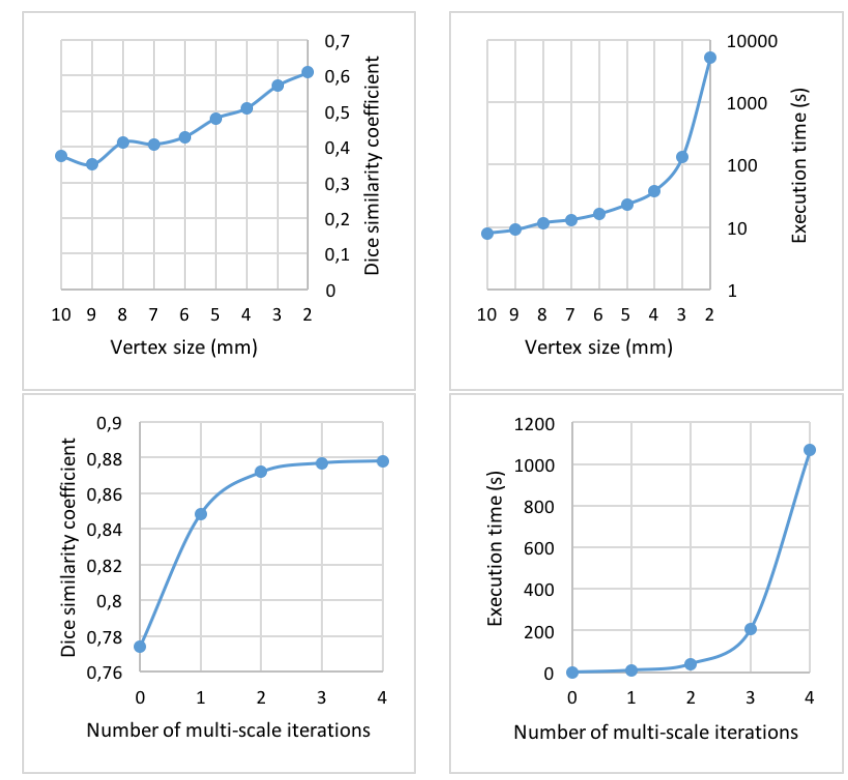

Fig. 3. Evolution of DICE coefficient and execution time with the vertex size and the number of multiscale iterations.

\begin{tabular}{|l|c|c|}
\hline DICE & Pulmonary & Aorta \\
\hline min & 0.8569 & 0.9393 \\
\hline mean & 0.8671 & 0.9585 \\
\hline max & 0.8751 & 0.9707 \\
\hline
\end{tabular}

Table 1. DICE similarity coefficents for the simulations.

\section{RESULTS AND DISCUSSION}

\subsection{Simulated dataset}

Simulated X-ray angiography images were generated using the XCAT motion simulator, which reproduces realistically both cardiac and respiratory motion. Those images, unlike clinical images, are perfectly calibrated and can only contain the desired vessel structure to ease the segmentation. Two sequences have been generated for validation purposes (77 pairs, 53 of pulmonary arteries and 24 of aortic arch). Results are obtained using a $\beta$ value of 1 .

As it is shown in figure 3, the accuracy and the execution time of our method are highly dependent on the initial size of the vertices and the number of multi-scale iterations. The size of the structure to reconstruct, as well as the resolution of the images are factors that also influence the choice of the size of the vertices and the number of multi-scale iterations. Initial size of $3 \mathrm{~mm}$ and two iterations of multiscale refinement was found to be a fair compromise between execution time and accuracy. Table 1 regroups the results of the method on our two generated sequences. Some false positives (i.e. vertices that should not be in the final reconstruction) are eliminated because of the distance ratio. However, some are still remaining due to overlapping. The results shown in figure 4 are a per- 


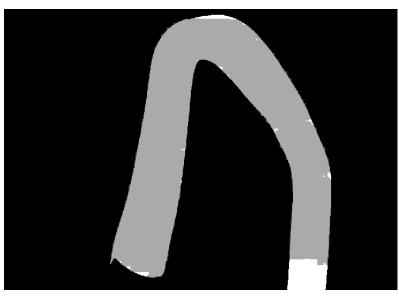

(a) LAT view

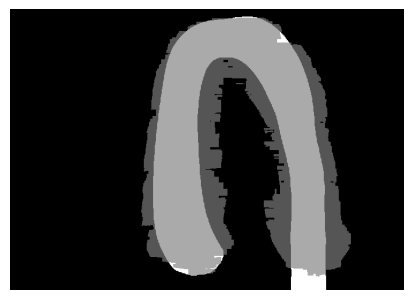

(b) Third view
Fig. 4. Reprojection of the volume. White, light gray and dark gray pixels represent, respectively, the initial segmentation, the accurate reprojection and the false positives.

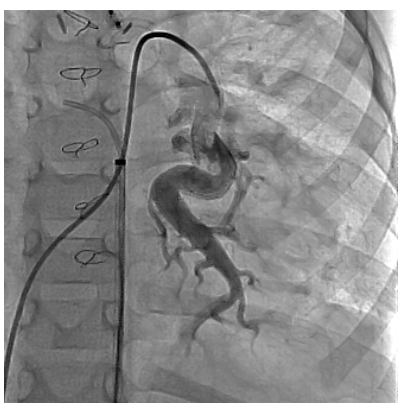

(a) PA view

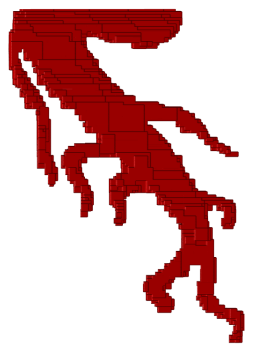

(b) $3 \mathrm{D}$ reconstruction
Fig. 5. 3D reconstruction of a part of a pulmonary artery from our clinical dataset.

fect illustration of the remaining false positives. In this third view, located between the two used for the $3 \mathrm{D}$ reconstruction, the DICE similarity measure is lower. Indeed, limited structural information lies in only two views. To obtain a volume closer to the reality, additional views must be used. Figure 2 presents graphical results of the reconstruction of an aorta.

\subsection{Clinical dataset}

Concerning the clinical validation, a small dataset of three pairs of images, taken from the same patient, has been used. Graphical results are shown in figure 5. A mean DICE similarity coefficient of 0.84 has been obtained, which promises for clinical data. These results are consistent with simulated results. This can be explained by the nature of the images used for the reconstruction. Indeed by using segmentation masks, we consider that the prior segmentation is accurate enough, which tends to make our method unaffected by noise and poor contrast.

\section{CONCLUSION}

We have proposed a novel graph-based voxel coloring approach for 3D reconstruction of vascular structures from only a single pair of angiographic views. Further works will consider incorporating shape prior to the reconstruction and cardiorespiratory motion compensation.

\section{REFERENCES}

[1] J. Hadida, C. Desrosiers, and L. Duong, "Stochastic $3 \mathrm{~d}$ motion compensation of coronary arteries from monoplane angiograms," in International Conference on Medical Image Computing and Computer-Assisted Intervention. Springer, 2012, pp. 651-658.

[2] R. Szeliski, Computer Vision: Algorithms and Applications, Springer, 2010.

[3] S. Seitz and C. Dyer, "Photorealistic scene reconstruction by voxel coloring," International Journal of Computer Vision, vol. 35, no. 2, pp. 151-173, 1999.

[4] R. Cardenes, A. Novikov, J. Gunn, R. Hose, and A. Frangi, "3d reconstruction of coronary arteries from rotational x-ray angiography," in 9th IEEE International Symposium on Biomedical Imaging (ISBI), 2012, pp. 618-621.

[5] S. Habert, N. Dahdah, and F. Cheriet, "A novel method for an automatic $3 \mathrm{~d}$ reconstruction of coronary arteries from angiographic images," in IEEE Information Science, Signal Processing and their Applications (ISSPA), 11th International Conference on, 2012, pp. 484-489.

[6] L. Grady, "Random walks for image segmentation," IEEE Trans. Pattern Anal. Mach. Intell., vol. 28, no. 11, pp. 1768-1783, Nov. 2006.

[7] E. Vachon, J. Miro, and L. Duong, “Online c-arm calibration using a marked guide wire for $3 \mathrm{~d}$ reconstruction of pulmonary arteries," in Proc. SPIE Medical Imaging, 2017.

[8] A. Frangi, W. Niessen, K. Vincken, and M. Viergever, "Multiscale vessel enhancement filtering," in International Conference on Medical Image Computing and Computer-Assisted Intervention. Springer, 1998, pp. 130-137.

[9] R. Hartley and A. Zisserman, Multiple view geometry in computer vision, pp. 239-261, Cambridge University Press, 2003.

[10] F. M'hiri, L. Duong, C. Desrosiers, and M. Cheriet, "Vesselwalker: Coronary arteries segmentation using random walks and hessian-based vesselness filter," in IEEE 10th International Symposium on Biomedical Imaging, 2013, pp. 918-921. 